\title{
Quantification of structural robustness: application to the study of a prestressed concrete beam
}

\author{
N.C. Kagho-Gouadjio ${ }^{1}$, A.D. OrCesi ${ }^{1, a}$, C.F. Cremona ${ }^{2}$ and C. Marcotte ${ }^{3}$ \\ 1 University Paris-Est, IFSTTAR, Materials and Structures Department, Champs-sur-Marne, France \\ 2 CEREMA/DTecITM, Technical center for Bridge Engineering, Sourdun, France \\ 3 CEREMA/DTerNP, Bridge Division, Haubourdin, France
}

Received 26 June 2013, Accepted 5 July 2014

\begin{abstract}
The term of "structural robustness" gives rise to various definitions and applications. In particular, the European structural standards Eurocodes recommend a structural design to be sufficiently safe against accidental or abnormal loads not explicitly considered in the design. This paper presents a probabilistic approach for the quantification of structural robustness, which measures the impact of a localized failure on the global structural failure. In order to identify the probabilistically most dominant failure mechanisms starting from a local failure, failure tree methods are used, such as the branch and bound method, the $\beta$-unzipping method, and an original approach combining the concepts of $\beta$ unzipping with some bounding aspects. These methods are used to identify dominant failure paths within reasonable computational times. In particular, it is possible to determine the failure path associated with the largest probability of failure, also called the reference path. Considering this reference path, some robustness metrics are proposed to quantify the relative magnitude (expressed in probabilities or in risk measures) between local and global failures. The proposed approach is applied to the structural analysis of a prestressed beam. The results obtained with the three methods as well as the computational times required each time are finally compared.
\end{abstract}

Key words: Structural robustness / risk / failure / robustness metrics

\section{Introduction}

In the field of structural engineering, the Eurocodes standards define structural robustness as "the ability of a structure to withstand events like fire, explosions, impact or the consequences of human error, without being damaged to an extent disproportionate to the original cause". This definition highlights the notions of initial damage (local failure) and disproportionate damage (global failure) [1-3]. With such a definition, an essential step is to characterize the transition of the system from a local damage (initial failure) to a global one (disproportionate failure). Several examples of structural failures illustrate the impact of local damage on global behavior such as the progressive and partial collapse of the Ronan Point tower in London (UK) in 1968 due to an internal gas explosion at the 18th floor, killing 4 people [4] or more recently, the I-35W Bridge collapse in Minneapolis (USA) in 2007 due to a design flaw, killing 13 people and injuring 145 [5] (Fig. 1).

\footnotetext{
${ }^{a}$ Corresponding author: andre.orcesi@ifsttar.fr
}

When exposed to an accidental action, a structure might loose one or more structural element(s) by failure. The structure is then said to be vulnerable and the degree of vulnerability depends on the extent of the observed direct consequences (damage immediately due to the action of accidental loads). Robustness is estimated taking into account the direct and indirect consequences due to the occurrence of a hazard [6-8]. A simple example is that an impact can cause the failure of a column which is a key element in the structural design (direct consequence). This local failure might then cause the progressive failure of other structural elements until the overall structural failure (indirect effect). Assessing the structural robustness leads to appreciate the gap between the local and the global damage of the system (Fig. 1).

With the aim of taking into account multiple uncertainties associated with loads, material and structural properties, this paper proposes a reliability-based approach to quantify structural robustness of civil engineering structures, but also tempts to introduce some "robustness" metrics to quantify the ability of the system to sustain local damage. To analyze structural robustness 

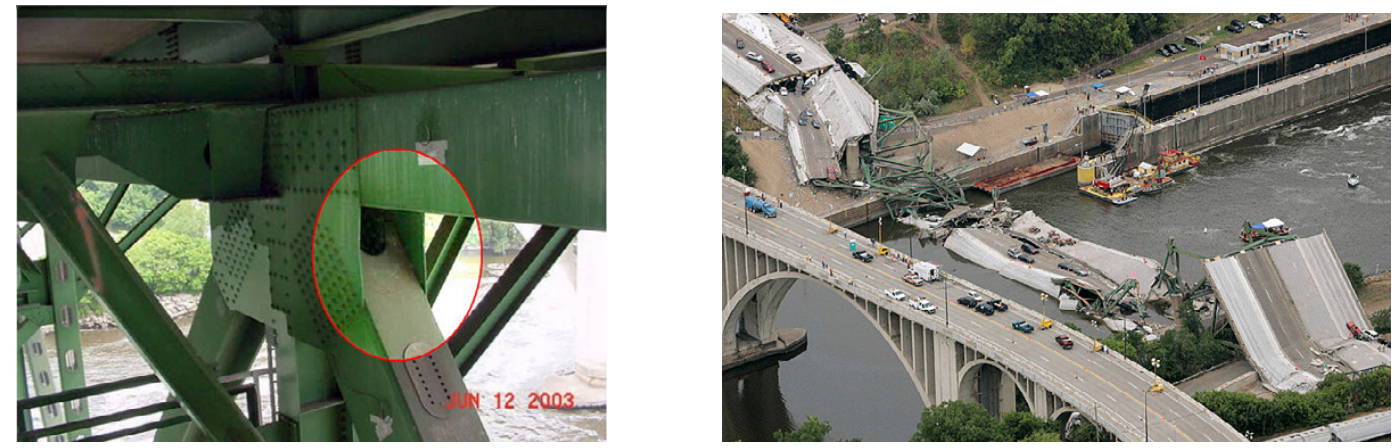

Fig. 1. Collapse of the Mississipi bridge, Minneapolis, USA, 2007.

in a general framework, the structural system should be firstly defined and modeled. Secondly, the local failure and the global failure should be well characterized to allow the identification of the probabilistically dominant complete failure paths. The local failure may occur at a structural element, a subset of structural elements or a critical area (failure mode). The global failure refers to a critical function of the structural system, which is no longer fulfilled, following the occurrence of a number of localized damages.

To determine the failure path with the largest probability of occurrence starting from the failure of one element, several failure path methods are used. The probabilistically dominant complete failure paths are identified using structural system reliability techniques such as the branch-and-bound method [9], the $\beta$-unzipping method [9] and an original hybrid $\beta$-unzipping/bounding approach [10-12]. Based on the failure path with the largest probability of occurrence (reference path), several probabilistic robustness metrics can be proposed, first by comparing the probability of the system to transit from local to global damage, and secondly by taking into account the notion of risk $[7,8]$, and measuring the gap between the consequences of local and global damage. The study of a prestressed beam is carried out to illustrate the proposed methodology.

\section{Metrics of structural robustness}

Two robustness metrics are introduced in this section, with the aim of determining the extent between local and global damage, as mentioned in the introduction. The first proposed robustness metrics is a ratio between local and global failure probabilities, as expressed below

$$
I_{r, 1}=1-\frac{P_{\text {global }}}{P_{\text {local }}}
$$

where $P_{\text {local }}=$ probability of local failure, and $P_{\text {global }}=$ probability of occurrence of the reference failure path. $I_{r, 1}$ varies between the interval [0;1[, the lower and upper bounds indicating non robust and robust structures, respectively. The second robustness metrics is expressed as

$$
I_{r, 2}=\frac{C_{\text {local }} P_{\text {local }}}{C_{\text {local }} P_{\text {local }}+C_{\text {global }} P_{\text {global }}}
$$

where $C_{\text {local }}=$ direct consequences associated with the initial damage and $C_{\text {global }}=$ indirect consequences associated with the system failure at a global scale [7]. The robustness metrics $I_{r, 2}$ compares the risk (defined herein as the multiplication of the consequence of one scenario by its probability of occurrence) at the local and global scale. This metrics can be rewritten as:

$$
I_{r, 2}=\frac{P_{\text {local }}}{P_{\text {local }}+a P_{\text {global }}}
$$

where $a=C_{\text {global }} / C_{\text {local }} \geq 1$ is a ratio between local and global consequences. It is interesting to note that $I_{r, 2}$ can be derived from $I_{r, 1}$ by the following expression

$$
I_{r, 2}=\frac{1}{1+a\left(1-I_{r, 1}\right)}
$$

As well as $I_{r, 1}$, values of $I_{r, 2}$ close to 0 and 1 indicate non robust and robust structures, respectively.

In the following of this paper, some notions and formulations of the reliability approach are used and coupled with the structural analysis. Some of these concepts are briefly reminded in this paragraph. In particular, a finite set of $n_{v}$ random variables $\mathbf{Z}=\left(z_{1}, \ldots, z_{n}\right)$ and a performance function $M$ are introduced and defined as $M=g(\mathbf{Z})$ where $g(\mathbf{Z})<0$ for $\mathbf{Z}$ in failure set, $g(\mathbf{Z})>0$ for $\mathbf{Z}$ in safe set, and $g(\mathbf{Z})=0$ for $\mathbf{Z}$ on the limit state surface [13]. The first-order reliability method (FORM) is used in the following calculations to approximate the probability of failure $P_{f}=P(g(\mathbf{Z}) \leq 0) \approx \Phi(-\beta)$ where $\beta$ is the reliability index [14] and $\Phi$ the standard normal cumulative distribution function.

An interpretation of the robustness metrics $I_{r, 1}$ and $I_{r, 2}$ is proposed thereafter considering a failure path noted $q$ (reference path), which can be for example the probabilistically most dominant failure path. The path $q$ is associated with the sequential order of failure elements that failed $q_{1}, q_{2}, \ldots, q_{n}$, where $q_{i}=i$ th failure mode of the path $q$ and $n=$ length of $q$. It is then possible to characterize local failure with the probability of failure of the first element in the reference path $q$. Thus $P_{\text {local }}=P_{f}\left(q_{1}\right)=P\left(g\left(q_{1}\right) \leq 0\right)$, with $P_{f}\left(q_{1}\right)=$ probability of failure of element $q_{1}$ and $g\left(q_{1}\right)=$ performance function associated with failure mode $q_{1}$. Assuming at least two failure events to reach the global failure, it is 
(a)

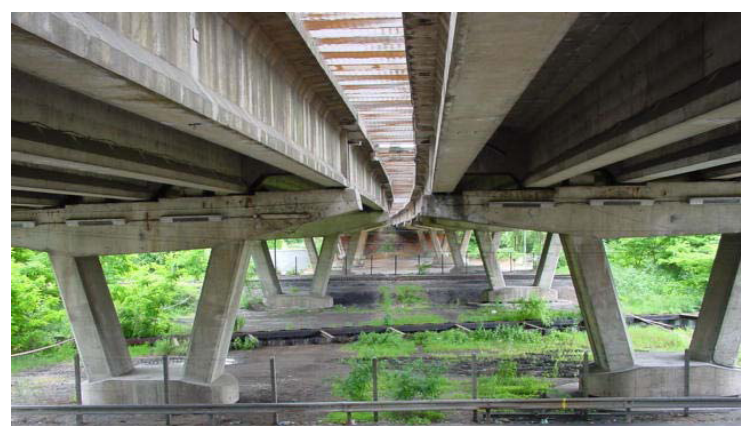

(b)

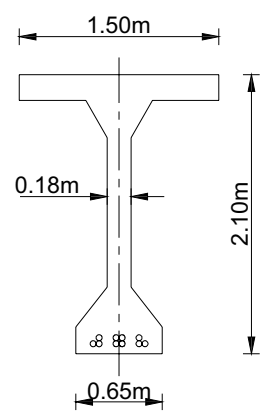

Fig. 2. (a) Merlebach viaduct and (b) cross-section of a beam at mid-span.

also possible to characterize the probability of global failure as $P_{\text {global }}=P\left(g\left(q_{1}\right) \leq 0 \bigcap_{i=2}^{n} g^{q_{1}, \ldots, q_{i-1}}\left(q_{i}\right) \leq 0\right)$ with $g^{q_{1}, \ldots, q_{i-1}}\left(q_{i}\right)=$ performance function associated with the failure mode $q_{i}$ knowing that failure modes $q_{1}, q_{2}, \ldots, q_{i-1}$ have already occurred (for $i>1$ ), respectively [8].

It is then possible to write the robustness metrics $I_{r, 1}$ as follows

$$
I_{r, 1}=1-\frac{P\left(g\left(q_{1}\right) \leq 0 \bigcap_{i=2}^{n} g^{q_{1}, \ldots, q_{i-1}}\left(q_{i}\right) \leq 0\right)}{P\left(g\left(q_{1}\right) \leq 0\right)}
$$

which is equivalent to perform the calculation of a conditional probability as below

$$
I_{r, 1}=1-P\left(\bigcap_{i=2}^{n} g^{q_{1}, \ldots, q_{i-1}}\left(q_{i}\right) \leq 0 \mid g\left(q_{1}\right) \leq 0\right)
$$

In case where the robustness metrics $I_{r, 2}$ is used, the expression becomes

$$
I_{r, 2}=\frac{1}{1+a \times P\left(\bigcap_{i=2}^{n} g^{q_{1}, \ldots, q_{i-1}}\left(q_{i}\right) \leq 0 \mid g\left(q_{1}\right) \leq 0\right)}
$$

\section{Application to the study of a prestressed concrete beam}

In this section, the proposed approach is applied to one of the beams of the Merlebach's VIPP [15]. VIPP are simple span viaducts of precast concrete girders prestressed by post-tension. Figure 2 a gives a representation of Merlebach's VIPP, which includes two decks consisting of six isostatic spans of length $32.50 \mathrm{~m}$. Spans of each deck contained five beams each (2.10 m height) spaced $3.15 \mathrm{~m}$ and interconnected by a slab of $1.65 \mathrm{~m}$ wide and $0.18 \mathrm{~m}$ thick. In the beams, the longitudinal prestressing was characterized by 10 STUP cables of cross section characteristics $12 \varnothing 8$ (Fig. 2b), whose the first six were anchored in the butt [15-17].
In this case study, structural robustness is assessed by analyzing crack propagation from a local scale to a global scale of the beam. Explicitly, the local failure is assumed to be the height of a cracked area in the heel of the beam exceeding a critical threshold, and the global failure is assumed to be the total volume of cracked concrete exceeding a pre-defined threshold [12]. The considered beam is simply supported at its two ends, and subjected to a static concentrated load $P$ at mid-span. Modeling of the structure is performed using (c) SETRA-ST1 software (Fig. 3a) which is a calculation software of bar structures [18]. This study presents the modeling of the VIPP beam as developed within the framework of an earlier study on the structure of the Merlebach's VIPP [15] that aimed to take into account the cracking in calculating isostatic prestressed concrete beam and to better understand the deformation of the beam operating in degraded mode.

The model in Figure 3a represents the prestressed cables in red, the neutral axis of the beam in black, and the studied area (detailed in Figs. 3b and 3c) in yellow. Modeling of the beam is affected such that it comprises three areas, the two zones at the ends of a length of $15.25 \mathrm{~m}$, each consisting of one bar, and the central zone of $2 \mathrm{~m}$, consisting of 20 bars (Fig. 3b). To characterize the damage path associated with crack propagation when a concentrated load $P$ is applied at mid-span, the area of interest is located at mid-span in the central zone (where crack initiation is the most probable) and comprises seven studied sections numbered 1 through 7 (Fig. 3c). These seven sections are considered to analyze the application of the proposed methodology in link with the definitions of local and global failures.

\subsection{Modeling of failure}

The local failure is assumed to be reached when the crack height $h_{i \text {, crack }}$ in the lower fiber of section $i$ is larger

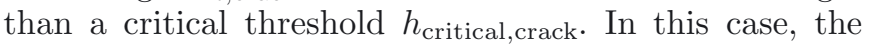
geometry of the beam is changed in this section by reducing the heel's height of an inclusive thickness in the lower part, which affects the moment of inertia in this particular section. Besides, the global failure considered 
Table 1. Statistics of random variables.

\begin{tabular}{|c|c|c|c|c|c|c|c|c|c|c|c|c|}
\hline Variables & $P$ & $\rho$ & $\sigma_{\text {cables }}$ & $\sigma_{\text {trac }}^{h}$ & $\sigma_{\text {trac }}^{1}$ & $\sigma_{\text {trac }}^{2}$ & $\sigma_{\text {trac }}^{3}$ & $\sigma_{\text {trac }}^{4}$ & $\sigma_{\text {trac }}^{5}$ & $\overline{\sigma_{\text {trac }}^{6}}$ & $\sigma_{\text {trac }}^{7}$ & $h_{\text {crack,critical }}$ \\
\hline Distribution & & mal & \multicolumn{10}{|c|}{ Lognormal } \\
\hline Mean & 0.79 & 2.5 & 800 & 3.27 & 3.27 & 3.27 & 3.27 & 3.27 & 3.27 & 3.27 & 3.27 & 0.05 \\
\hline$(\mu)$ & $\mathrm{MN}$ & t.m $\mathrm{m}^{-3}$ & $\mathrm{MPa}$ & $\mathrm{MPa}$ & $\mathrm{MPa}$ & $\mathrm{MPa}$ & $\mathrm{MPa}$ & $\mathrm{MPa}$ & $\mathrm{MPa}$ & $\mathrm{MPa}$ & $\mathrm{MPa}$ & $\mathrm{m}$ \\
\hline $\mathrm{COV}$ & $5 \%$ & $5 \%$ & $9 \%$ & $20 \%$ & $20 \%$ & $20 \%$ & $20 \%$ & $20 \%$ & $20 \%$ & $20 \%$ & $20 \%$ & $3 \%$ \\
\hline
\end{tabular}

(a)

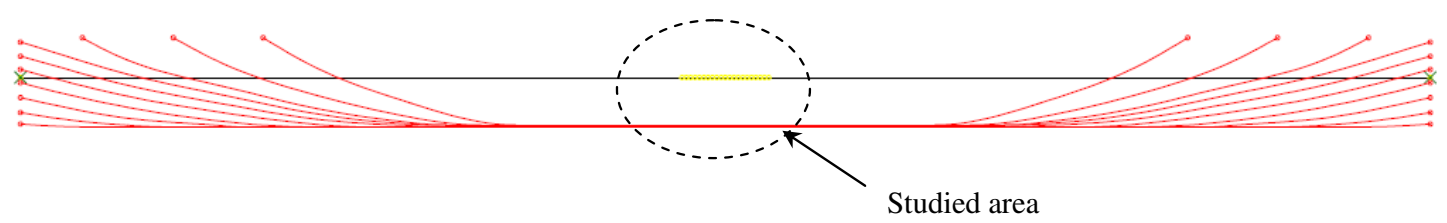

(b)

\begin{tabular}{|c|c|c|c|}
\hline$\triangle$ & $15.25 \mathrm{~m}$ & $2.00 \mathrm{~m}$ & $15.25 \mathrm{~m}$ \\
\hline & $\begin{array}{c}\text { Zone } 1 \\
1 \text { beam element }\end{array}$ & $\begin{array}{c}\text { Zone } 2 \\
20 \text { beam elements }\end{array}$ & $\begin{array}{c}\text { Zone } 3 \\
1 \text { beam element }\end{array}$ \\
\hline
\end{tabular}

(c)

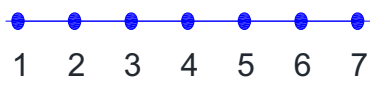

Fig. 3. (a) Modeling of the beam, (b) studied areas, and (c) detail of the seven sections in zone 2.

herein is reached when the volume of cracked concrete $v_{\text {crack }}$ calculated in the seven studied sections (see Fig. 3) as:

$$
v_{\text {crack }}=e_{\text {section }} l_{\text {heel }} \sum_{i=1}^{7} h_{i, \text { crack }}
$$

is larger than a critical threshold $v_{\text {critical,crack, where }}$ $e_{\text {section }}=0.10 \mathrm{~m}$ is the spacing between the studied sections and $l_{\text {heel }}=$ the width of the heel. In the following, $h_{\text {critical,crack }}$ is assumed to follow a lognormal distribution with mean and coefficient of variation (COV) fixed at $0.05 \mathrm{~m}$ and $3 \%$, respectively. The mean value of the tension in the prestressing cables $\sigma_{\text {cables }}$ is furthermore taken to be $800 \mathrm{MPa}$ [12]. The statistics of random variables used are provided in Table 1 , where $\sigma_{\text {trac }}^{h}=$ limit stress of the concrete in tension outside the study area, $\sigma_{\text {trac }}^{i}=$ limit stress of the concrete in tension in the study area, for the considered section $i$. Finally, the volume of cracked concrete $v_{\text {crack}}$, which is calculated along the failure path by considering each time the design point values of the parameters in Table 1 when the limit state for critical height is reached (local failure), is compared to the

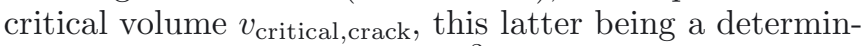
istic parameter fixed at $0.04 \mathrm{~m}^{3}$.

\subsection{Structural robustness analysis}

The results obtained by leading the three event-tree approaches are presented in this section and detailed in Table 2 and in Figure 4. The parameter $a$ is fixed at 100 in this section for illustration. Such a parameter represents the ratio between local and global consequences, which can be understood in this example as the difference of required maintenance budget when the first crack is observed and when a critical volume of cracked concrete is reached and a major rehabilitation of the beam is required. The computations of this paper are performed using a workstation with dual quad core Intels Xeons processors and 3.5 GB of RAM.

It appears in this example that the two methods based on $\beta$-unzipping are faster to converge compared to the branch and bound method. In this example, there is not a significant gain in calculation times when the bounding process is activated in addition to the $\beta$-unzipping even if the number of complete failure paths is significantly reduced (compare Figs. $4 \mathrm{~b}$ and $4 \mathrm{c}$ ). Also, these results show that the $\beta$-unzipping and the $\beta$-unzipping with bounding methods identify the same probabilistically most dominant failure path 4-5-3-2-6, with a failure probability $P_{f}=3.97 \times 10^{-1}$. The branch and bound method identifies the reference path $4-7$ which is associated with a larger failure probability $P_{f}=4.20 \times 10^{-1}$.

It is noted that both methods using $\beta$-unzipping concepts lead to a symmetric failure path (4-5-3-2-6) with local failures progressively extending from the center of the studied area (where the load is applied) to the extremities of this area. Conversely, the reference failure path obtained with the branch and bound method straightly moves from Sections 4 to 7 . The difference in those paths can be explained by the fact that whereas the branch and bound considers the probability of failure of the entire path at each node of the tree in Figure 4, the methods using $\beta$-unzipping concepts consider at each node the probability of failure of the last element in the failure 
Table 2. Obtained results.

\begin{tabular}{cccccc}
\hline $\begin{array}{c}\text { Load } P \text { acting at } \\
\text { section } 4 \text { (mid-span) }\end{array}$ & $I_{r, 1}$ & $I_{r, 2} a=100$ & Reference path & $\begin{array}{c}\text { Probability of failure } \\
\text { of the reference path }\end{array}$ & CPU time (s) \\
\hline $\begin{array}{c}\text { Branch and bound } \\
\beta \text {-unzipping }\end{array}$ & 0.21 & 0.01 & $4-7$ & $4.20 \times 10^{-1}$ & 55500 \\
$\beta$-unzipping with bounding & 0.25 & 0.01 & $4-5-3-2-6$ & $3.97 \times 10^{-1}$ & 16000 \\
\hline
\end{tabular}

(a)

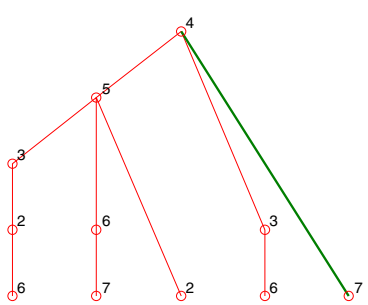

(c)

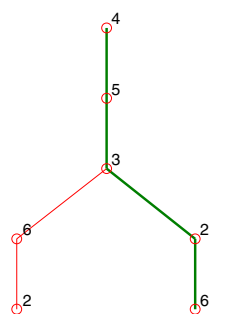

(b)

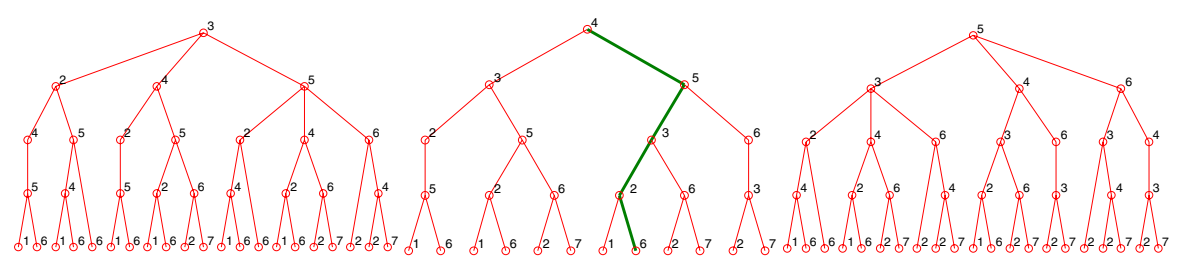

Fig. 4. Dominant failure paths obtained with (a) branch and bound method, (b) $\beta$-unzipping method, and (c) $\beta$-unzipping with bounding method.

(a)

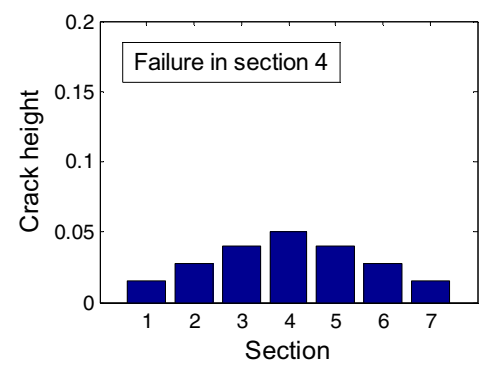

(b)

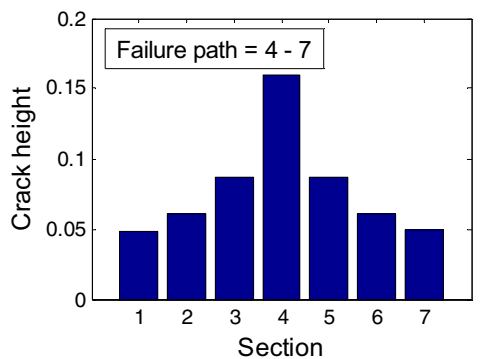

(c)

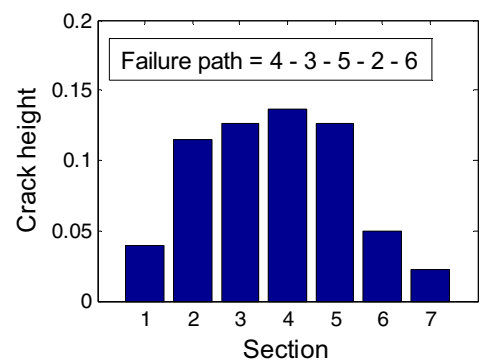

Fig. 5. Crack heights in each of the seven sections for the failure path (a) 4, (b) 4-7, and (c) 4-3-5-2-6.

path. These two paths, even if different, lead to a volume $v_{\text {crack }}$ larger than the accepted threshold $v_{\text {critical,crack. }}$ Finally, the obtained robustness metrics $I_{r, 1}$ and $I_{r, 2}$ are very close with the three different methods. It is observed that using the ratio $a$ for $I_{r, 2}$ leads to values much closer to 0 than those obtained with the definition of $I_{r, 1}$. If the focus is put on the probability of the system to transit from local to global damage with $I_{r, 1}$, it is also possible to integrate the notion of risk with $I_{r, 2}$ by considering the gap between the consequences of local and global damage. In this sense, both metrics are complementary and their values should not be directly compared. To further illustrate the results, Figures $5 \mathrm{a}, 5 \mathrm{~b}$ and $5 \mathrm{c}$ show the crack heights in each of the seven sections of the studied area when 4, 4-7, and 4-3-5-2-6 have failed, respectively. In these figures, the values of the variables are those obtained at the design point when the limit state for critical height is reached (local failure) in the last element of the failure path. It is observed that the profile of cracks is more concentrated in the center of the studied area in Figure 5b whereas it is more spread in Figure 5c. These results are in accordance with the difference in the length of the failure path itself. For the failure path 4-7, the global failure is quickly reached and is associated with a sharp and extended crack in Section 4. For the failure path 4-3-5-2-6, the failure spread along the different sections which present homogeneous crack heights. Considering the robustness metrics, the path 4-7 is obviously associated with the most critical value.

\section{Conclusions}

Design for robustness represents a scientific and technical challenge for civil engineers. A major problem with regard to its incorporation into current methods of design and management is the ability to quantify it. The work presented in this paper proposes a probabilistic approach for the quantification of structural robustness. The 
proposed approach is based on the study of a series of failure propagation in the structure, to identify the probabilistically most significant global failures and to derive a gap between the probabilities of local and global damage. This approach has been applied in the case of a prestressed concrete beam, which shows the application of the proposed methodology when the concepts of local and global failures can be well characterized and modeled. Further reflection is obviously necessary on the operational implementation of the proposed robustness metrics, and their inclusion in a regulatory framework. Also, a thorough sensitivity analysis still needs to be performed to assess how the results of the proposed model are apportioned to the different sources of uncertainty in its inputs, and to the discretization of the failure propagation.

\section{References}

[1] EN 1990, Basis of Structural design. Eurocode 0: CEN, 2002

[2] EN 1991-1-7, Actions on Structures: Part 1-7: Accidental Actions. Eurocode 1: CEN, 2006

[3] H. Gulvanessian, T. Vrouwenvelder, Robustness and the Eurocodes, Structural Engineering International (2006)

[4] Ministry of Housing and Local Government, Report of the Inquiry into the Collapse of Flats at Ronan Point, Canning Town, HM Stationery Office, London, 1968

[5] National Transportation Safety Board, Collapse of I35W Highway Bridge, Minneapolis, Minnesota, Highway Accident Report NTSB/HAR-08/03, Washington, DC, 14. USA, 2008

[6] M.H. Faber, M.A. Maes, D. Straub, J. Baker, On the Quantification of robustness of Structures, Offshore Mechanics and Arctic Engineering, Proc. Intern. Conf., Hamburg, Germany, 2006
[7] J.W. Baker, M. Schubert, M.H. Faber, On the assessment of robustness, Elsevier, Struct. Saf. 30 (2008) 253-267

[8] C. Cremona, Structural performance - Probability-based assessment, Wiley \& Sons, 2011, 448p.

[9] P. Thoft-Christensen, Y. Murotsu, Application of structural Systems Reliability Theory, Springer-Verlag, Berlin, 1986

[10] N. Kagho, A. Orcesi, C. Cremona, Robustness analysis of structural systems considering accidental actions, ICASP11, 2011

[11] N. Kagho, A. Orcesi, C. Cremona, A probabilistic approach for the quantification of structural robustness, IABMAS2012, Stresa, Italy, 2012

[12] N. Kagho, Étude de la vulnérabilité et de la robustesse des ouvrages, Ph.D thesis, University of Paris-est, France, 2013

[13] H.O. Madsen, S. Krenk, N.C. Lind, Methods of structural safety. Englewood Cliffs (NJ), Prentice-Hall, Inc, 1986

[14] R.E. Melchers, Structural Reliability Analysis and Prediction, John Wiley \& Sons. Chichester, 2nd edn, 1999

[15] C. Cremona, Suivi du comportement mécanique d'une poutre de VIPP sous chargement, le cas du VIPP de Merlebach, LCPC, 2007

[16] C. Tessier, L. Gaillet, D. Bruhat, R. Michel, S. Noblet, V. Barbier, Les moyens d'auscultation et de surveillance : Applications au viaduc de Merlebach, Journées CABLES2005, Nantes, 2005

[17] S. Mohammadkhani-Shali, Contribution à l'étude de la redondance dans les ponts: analyses des mécanismes de défaillance par surfaces de réponse, Ph.D thesis, ENPC, 2007

[18] Sétra, ST1, logiciel de Calcul de structures, version 22, 2011 